\title{
Mathematical approach to the psychological aspects of human
}

\author{
2020/12/24 4th Edition \\ Takuya Yabu(takuya.yabu@live.jp)
}

\begin{abstract}
Belonging to a group, there are people who are always bullied no matter how they behave. They don't know how to treat their own emotions. So, I considered human emotions. I mathematically modeled human emotion processing in two stages: what kind of emotions we receive from events and how we react from the emotions we receive. The part that receives emotions from events and the part that responds from emotions are modeled by a one-dimensional random walk or Wiener process, and the distribution of individual emotions is represented by a fixed probability distribution. Therefore, I also showed that when individuals gather to form a group, the distribution of emotions is also represented by a fixed distribution. In addition, I showed as application examples of these models, the nature of events, the meaning of emotional distribution, and how to read the air, and how to deal with one's character, and how to show one's reaction and what to do for events which have a large magnitude.
\end{abstract}

\section{Introduction}

Belonging to a group, there are people who are always bullied no matter how they behave. They are bullied, whether they behave like them or apologize as soon as they feel a little bad. They don't know how to treat their own emotions. So I considered human emotions.

Mathematical approach to decisions of human has been researched. The decision field theory mathematically explained how human decide things (Busemeyer \& Townsend, Decision Field Theory: A dynamic cognition approach to decision making, 1993) (Busemeyer \& Diederich, Survey of decision field theory, 2002). It used to be applied to static modeling, but in recent years, it has been actively applied to dynamic modeling (Hancock, Hess, \& Choudhury, 2019) and research on quantum decision-making (XIN, LI, BI, \& YAN , 2019). Not limited to the decision field theory, mathematical models (Chamberlin, 2019) are actively applied not only to psychology but also to other fields. It has been applied to dynamic psychological events (Cox \& Criss, 2020) and to econometrics (Hancock T. O., 2019). There is also an application of mathematical models in experimental psychology (Bolger, Zee, Rossignac-Milon, \& Hassin, 2019). Bayesian modeling has also been applied to psychology (Burigana \& Vicovaro, 2020) (Neufeld \& 
Cutler , 2019). But as far as the author knows, it doesn't exist about mathematical approach to the psychological aspects of human, like emotion processing, human character, human reaction to the events.

So, I thought about what I could say mathematically about the psychological aspects of human beings and what they should do if they couldn't handle it well.

2. About personal emotion processing

\subsection{About individual reaction functions}

Suppose that when an event $X$ occurs, the emotion $x$ occurs. In response to this, a reaction called $F(x)$ occurs. Figure 1 shows the relationship.

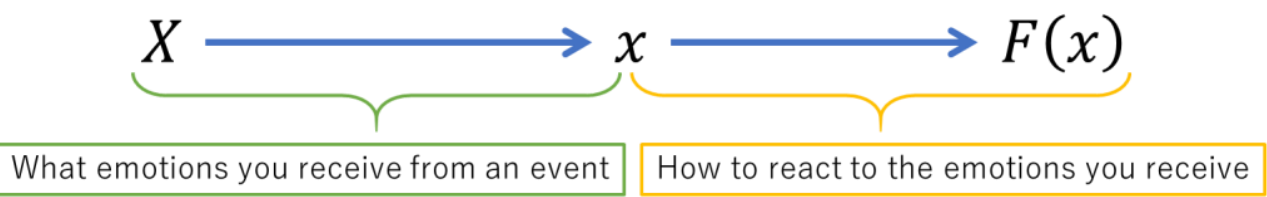

Figure 1 About the relationship between events, emotions, and reactions

Here, for $X$, which is the event and the reaction of oneself or the other party, you determine whether it produces positive or negative emotions for you, and whether it is emotionally or behaviorally large or small. Behavioral is because big behaviors tend to be when emotions are high, and small behaviors tend to be when emotions are low. As a result, the initial value of emotion $x$ is randomly determined by $-\infty<x<\infty$. From there, I think the individual randomly adds 1 or -1 to that value, aiming for $x=0$ for emotion $x, x=0$ is a convincing state for a series of events. In other words, a state of conviction is a state of stability, security, and emotionlessness. Toward that, we aim for $x=0$ by the chain of events or reactions to them and emotional reception. I think that this individual emotion processing is the character of the individual. Even if $x=0$ once, I think that we may continue to add 1 and -1 . It is a phenomenon that one worry oneself.

In other words, if you think about this process in reverse, it seems that emotion processing is performed by a one-dimensional random walk. With a probability of $\frac{1}{2}$, it is judged whether it is a positive emotion or a negative emotion, and it is considered that the total emotion is obtained by adding them together. At this time, from the one-dimensional random walk, the mean (expected value) $\mu$ of emotion $x$ is 0 , and the variance $\sigma^{2}$ is $n$.

Now, suppose that this emotion processing is performed $m$ times. At this time, the value of each emotion is $x_{i}(i=1, \cdots, m)$. From the central limit theorem, the following 
holds for a certain probability distribution $P$.

$$
\begin{gathered}
\lim _{m \rightarrow \infty} P\left(\frac{x_{1}+\cdots+x_{m}-m \mu}{\sqrt{m} \sigma} \leq \alpha\right)=\lim _{m \rightarrow \infty} P\left(\frac{\frac{x_{1}+\cdots+x_{m}}{m}-\mu}{\frac{\sigma}{\sqrt{m}}} \leq \alpha\right) \\
=\frac{1}{\sqrt{2 \pi}} \int_{-\infty}^{\alpha} e^{-\frac{x^{2}}{2}} d x
\end{gathered}
$$

Now consider $\sigma^{2} . \sigma^{2}=n_{1}+\cdots+n_{m}$, but it is thought that the value is dominated by the largest $n$. Therefore, when $n \rightarrow \infty$ exists, $\sigma^{2} \rightarrow n$. Also, this time $\mu=0$. Therefore,

$$
\lim _{m, n \rightarrow \infty} P\left(\frac{\frac{x_{1}+\cdots+x_{m}}{m}}{\frac{\sqrt{n}}{\sqrt{m}}} \leq \alpha\right)=\frac{1}{\sqrt{2 \pi}} \int_{-\infty}^{\alpha} e^{-\frac{x^{2}}{2}} d x
$$

Therefore, when $m, n \rightarrow \infty$, it can be regarded as $n \rightarrow m$, and the denominator $\frac{\sqrt{n}}{\sqrt{m}}$ approaches 1 from equation (2), so the following equation holds.

$$
\lim _{m, n \rightarrow \infty} P\left(\frac{x_{1}+\cdots+x_{m}}{m} \leq \alpha\right)=\frac{1}{\sqrt{2 \pi}} \int_{-\infty}^{\alpha} e^{-\frac{x^{2}}{2}} d x
$$

That is, the probability distribution of emotions' mean converges to the standard normal distribution. Therefore, the probability density function $p(x)$ of emotions' mean is expressed by the following equation.

$$
p(x)=\frac{1}{\sqrt{2 \pi}} e^{-\frac{x^{2}}{2}}
$$

The probability density function of an individual's emotions can be expressed by equation ( 4 ) if the emotion processing is repeated many times and they are continuously averaged.

I think that how an individual reacts is automatically determined by what happens and what emotions one receives. In other words, once the events and emotions are decided, the individual reaction is shown like a dictionary. Therefore, the individual reactions will be determined by how the events are happened and how emotions are determined.

Here, the event cannot be influenced by oneself. Individuals cannot influence what has happened. An individual can influence an event, whether given by others, from nature, or as one's own thoughts.

Also, an individual cannot influence whether one receives positive or negative emotions from an event. In other words, choosing the sign of emotion for event $X$ was the basis of an individual's character, but an individual can't control one's character. An 
individual only can change reactions.

The individual gives an initial value of emotion $x$ to $X$ which is the event and the reaction of oneself or the other party. The criterion is given by equation (4). Since there is a one-to-one correspondence between $X$ and $x$, there is a one-to-one correspondence between the absolute value of emotion and the magnitude of reaction. When one has a big positive emotion, one has a big reaction that is good for one. When one has a big negative emotion, one has a big reaction that is not good for one. When one has a small emotion, one has a small reaction.

\subsection{The variance of emotion processing}

The probability density function for a one-dimensional random walk is given by the following equation.

$$
p(x, n)=\frac{1}{\sqrt{2 \pi n}} e^{-\frac{x^{2}}{2 n}}
$$

For equation (5), consider the variance of $x$. Assuming that emotion processing is performed $m$ times, let $x_{j}(j=1, \cdots, m)$ and consider the following formula. Note that the average of $x_{j}$ is 0 here. Here, $\hat{\sigma}^{2}$ is an unbiased variance.

$$
\tilde{\chi}^{2}=\frac{x_{1}^{2}}{\frac{\hat{\sigma}^{2}}{(m-1)}}+\cdots+\frac{x_{m}^{2}}{\frac{\hat{\sigma}^{2}}{(m-1)}}=\frac{1}{\frac{\hat{\sigma}^{2}}{(m-1)}} \sum_{j=1}^{m} x_{j}^{2}
$$

In addition, the formula transformation continues.

$$
\tilde{\chi}^{2}=\frac{1}{\hat{\sigma}^{2}} \sum_{j=1}^{m} x_{j}^{2}=\frac{\frac{1}{\frac{\sigma^{2}}{(m-1)}} \sum_{j=1}^{m} x_{j}^{2}}{\frac{1}{(m-1)} \cdot \frac{(m-1) \hat{\sigma}^{2}}{\sigma^{2}}}
$$

That is, $\frac{\tilde{\chi}^{2}}{m-1}$ is an $F$ distribution with two degrees of freedom $m-1$.

Let us consider $\frac{\tilde{\chi}^{2}}{m-1}$ again from equation (6). When the number of times emotions are processed $m \rightarrow \infty$, the following formula holds.

$$
\begin{aligned}
& \frac{\tilde{\chi}^{2}}{m-1}=\frac{1}{m-1}\left(\frac{x_{1}^{2}}{\frac{\hat{\sigma}^{2}}{(m-1)}}+\cdots+\frac{x_{m}^{2}}{\frac{\hat{\sigma}^{2}}{(m-1)}}\right) \\
& =\frac{1}{m-1}\left(\frac{x_{1}^{2}}{\frac{(m-1)}{(m-1)}}+\cdots+\frac{x_{m}^{2}}{\frac{(m-1)}{(m-1)}}\right) \rightarrow \frac{x_{1}^{2}+\cdots+x_{m}^{2}}{m}
\end{aligned}
$$


In other words, $\frac{\tilde{\chi}^{2}}{m-1}$ is the variance of emotion $x$. Therefore, the variance $\sigma^{2}$ follows an $F$ distribution with two degrees of freedom (number of emotional processes) of $m-1$. The graph of $\sigma^{2}$ is as follows.

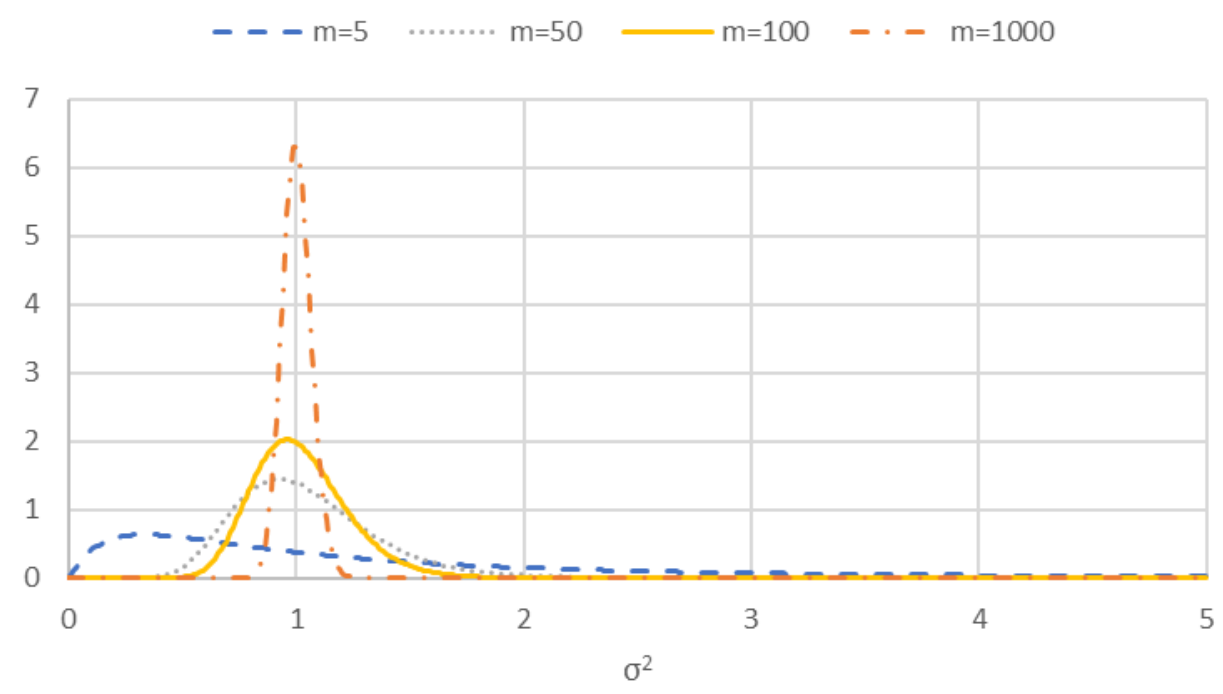

Figure 2 Graph of $\sigma^{2}(F$ distribution with two degrees of freedom $m-1)$

The mean and variance of the $F$ distribution with two degrees of freedom $m-1$ are:

$$
\text { Mean } \frac{m-1}{m-3} \text {, Variance } \frac{4(m-1)(m-2)}{(m-3)^{2}(m-5)}
$$

In other words, when $m \rightarrow \infty$, the mean is 1 and the variance is 0 . This is consistent with the variance $\sigma^{2}=1$ in the probability distribution of emotions' mean in Section 2.1 .

\subsection{From discrete to continuous}

Regarding the above, replace "adding $n$ times" with thinking for a certain period of time. Therefore, consider the following Wiener process. Here, $\beta$ is a positive real number and $W_{t}$ is a standard Brownian motion. $\beta$ is your whimsical ratio.

$$
x=\beta W_{t}
$$

Now, from equation ( 10 ), which is a stochastic differential equation, the following equation can be obtained. This is an equation called the Fokker-Planck equation. Where $p(x, t)$ is the probability density function.

$$
\frac{\partial p}{\partial t}=\frac{1}{2} \beta^{2} \frac{\partial^{2} p}{\partial x^{2}}
$$

Consider also from one-dimensional random walk. Let $P(x)$ be the probability of 
taking a certain emotion $x$. Now, suppose that emotions change by a small amount $a$. At this time, consider the following values

$$
-\{P(x+a)-P(x)\}
$$

This value represents the amount of change in the probability that emotions change, and is also the amount required to change a minute amount $a$. Consider the following formula as an amount considering how much it changes per unit time $\Delta t$.

$$
Q=-\frac{1}{\Delta t}\{P(x+a)-P(x)\}=-\frac{1}{\Delta t} \frac{d P}{d x} a
$$

This $Q$ represents the amount required to change a minute amount $a$ per unit time. From equation (13), the following equation is obtained with $p(x)=\frac{P(x)}{\mathrm{a}}$.

$$
Q=-\frac{a^{2}}{\Delta t} \frac{d p}{d x}
$$

Now, the idea is the same as the one-dimensional random walk. Here, considering how much $a$ is a value per unit amount of $x$, it is 2 because it takes a positive value and a negative value. That is, $a=\frac{1}{2}$. Also, since $\Delta t$ needs to obtain a for two times, this is also $\Delta t=\frac{1}{2}$. Therefore, the following equation can be obtained by substituting it into equation (14).

$$
Q=-\frac{1}{2} \frac{d p}{d x}
$$

Consider the time change of this $p$. The magnitude of $p$ per minute amount $d x$ of an emotion is $p d x$. Considering the amount of change over time, it is $\frac{\partial p}{\partial t} d x$. Considering how much $Q$ changes with respect to this $d x$, the following equation is obtained.

$$
Q(x)-Q(x+d x)=\frac{1}{2} \frac{\partial^{2} p}{\partial x^{2}} d x
$$

This value is equal to $\frac{\partial p}{\partial t} d x$. Therefore, the following partial differential equation is obtained.

$$
\frac{\partial p}{\partial t}=\frac{1}{2} \frac{\partial^{2} p}{\partial x^{2}}
$$

The partial differential equation (17) obtained from equation (1) and the Fokker-Planck equation ( 11 ) are the same equation. So you get:

$$
\beta^{2}=1, \beta=1
$$

This is the formula that $\beta$ satisfies.

From equation (17), the following equation is obtained. Here, $t$ is the time until the 
emotion processing is completed.

$$
p(x, t)=\frac{1}{\sqrt{2 \pi t}} e^{-\frac{x^{2}}{2 t}}
$$

From the discussion so far, the average distribution of emotions after many emotional processes is 0 on average and 1 on variance. Therefore, in equation (19), $t \rightarrow 1$.

3. About emotion processing when forming a group

3.1. About the reaction function of the group

Consider when individuals are grouped together. We consider the emotion processing of a group to be the average of the group. Then, of course, the probability density function of the group's emotions is equal to equation (4) and is given by:

$$
p_{\text {group }}(x)=\frac{1}{\sqrt{2 \pi}} e^{-\frac{x^{2}}{2}}
$$

The graph of equation (20) is as follows.

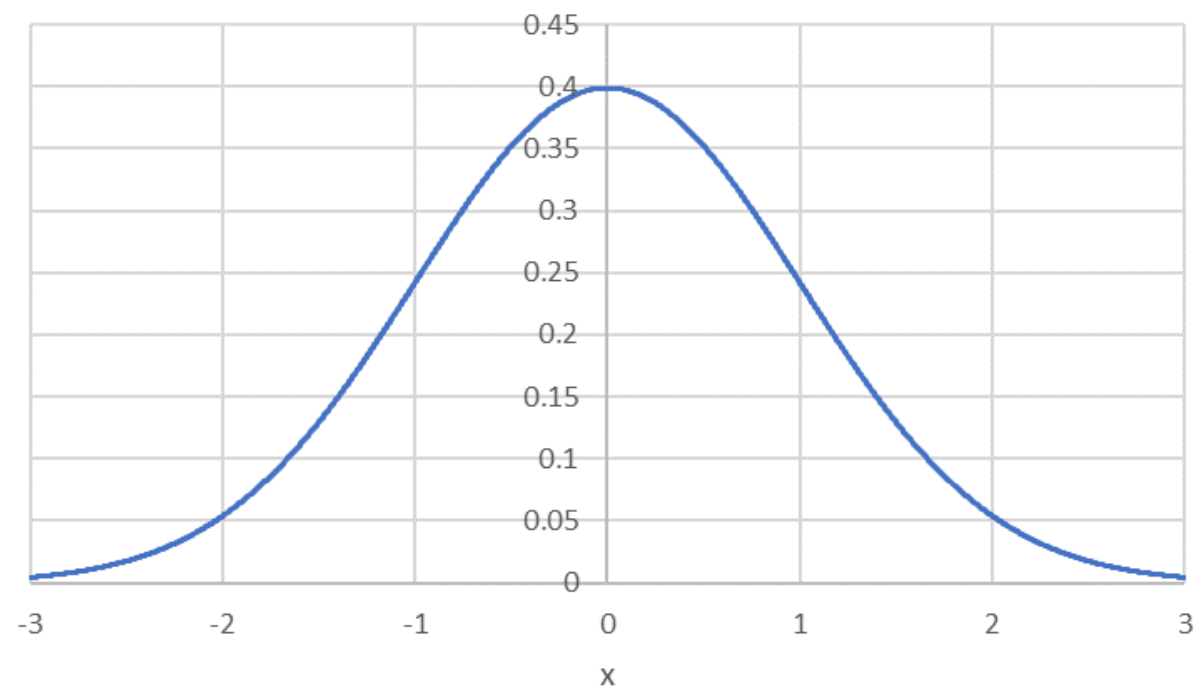

Figure 3 The probability density function of individual's and group's emotion

From Figure 3, it turns out that when humans are grouped together, they perform this kind of emotion processing.

\subsection{About emotion processing in a group}

From Section 3.1, we can see that the probability density function of individual's and group's emotion are equal. From this, it can be said that various things can be said. First, you don't have to think about your own boundaries. Since every individual has the same emotional probability density function and reaction function, What I hate is what 
the group hate, and What I like is what the group like. The only difference is whether you can read the air well or not. If you don't like it, you can say you don't like it.

Also, democracy is established as a political means because it is easy to select people who can read the air by majority vote, and they will execute the desired policy. In a tyranny, it is possible that a person who does not know how to read the air will become king due to his pedigree, and if that happens, it will not work well.

Furthermore, it can be said that a group functions as a group because the probability density function of individual's and group's emotion are equations (20) and (4). When many people get together, the probability density function of emotion is the same without doing anything, so control can be taken as a group. Therefore, people who do not know how to read the air stand out. They don't get used to the group. Therefore, it is thought that the problem of bullying and power harassment will occur if people who do not understand how to read the air are divided into those who attack and those who are attacked. On the other hand, most humans are indifferent because they do not know how to react to the reaction from people who do not understand how to read the air is different, or because they do not react not to disturb the group. Considering that the probability of not knowing how to read the air is small, it can be explained that bullying and power harassment occur in small groups. It is also possible that the attacker wants to atrophy most humans by becoming aggressive because he is not eliminated.

When three people get together, they may be divided into two and one, probably because there are two people who can read the air and one is a little weak at reading the air.

People who are always bullied have the same emotional probability density function as the neurotypical ones. In other words, it's just a small difference. Because the reaction of the people who are attacked is interesting to the people who attack, bullying and power harassment will probably occur.

3.3. About the characteristics of $X$ which is the event and the reaction of oneself or the other party

Consider the characteristics of $X$ which is the event and the reaction of oneself or the other party.

$X$ become smaller and forgotten over time. It is also likely to be forgotten or remembered. Therefore, consider a one-dimensional random walk with the value $\tau$ (unit is seconds, but 0 if it is 0 or less) minus the period judged to be important from the period judged to be insignificant. Derived using the central limit theorem and so on, the probability density function can be obtained by the following equation. 


$$
p(\tau)=\sqrt{\frac{2}{\pi}} e^{-\frac{\tau^{2}}{2}}, \tau \geq 0
$$

Therefore, for the emotion $x$ generated from $X$, which is the event and the reaction of oneself or the other party, the following equation can be obtained as a function of the event considering the initial value using equation (21).

$$
x=x_{0} e^{-\frac{\tau^{2}}{2}}, \tau \geq 0
$$

Where $x_{0}$ represents the initial value of the emotion.

That is, most individuals, and groups of individuals, forget about emotion $x$ in equation ( 22$)$.

Also consider that emotion $x$ is repeated $n$ times. In this case, the following formula holds.

$$
x=x_{0} e^{-\frac{\tau_{1}^{2}}{2}}+x_{0} e^{-\frac{\tau_{2}^{2}}{2}}+\cdots+x_{0} e^{-\frac{\tau_{n}^{2}}{2}}=n x_{0} e^{-\frac{\tau_{1}^{2}+\cdots+\tau_{n}^{2}}{2 n}}
$$

In other words, the larger $n$, the larger the value of the emotion and the harder it is to forget.

From this, if you repeat the same action or thinking about something, the value of emotion $x$ will increase in an individual or groups of individuals, and resulting in rejection or a big negative reaction to yourself. As mentioned in Section 6, you can think unconsciously as many times as you like in one second. If you give the same actions and thoughts, it is better to open the period and reduce the number of times as much as possible. Repeated actions and thoughts can lead to resentment and depression. Even repeating favorable behaviors can lead to disgusting and negative emotions, and the gradual addition of negative values can lead to large negative emotions as a whole, which can be rejected.

\subsection{Tips for worrying}

Human's $\beta$ is 1 , and the value does not change. And, due to the nature of the $F$ distribution, $t \rightarrow 1$. First, regardless of whether people are worried for a long time or for a short time, the probability density function of an individual's emotion does not change in equation (4), and the shape of the graph does not change from that given in Figure 3. Even if people worry for a long time, people's emotional range will not expand. Even if people worry for a short time, people's emotional range will not be narrowed. The stochastic process expressed by equation (10) is called the Ornstein-Uhlenbeck process (mean reversion process), and when the time $t$ to worry becomes longer, the emotion $x$ 
approaches the mean value 0 . In other words, the more people worry, the less positive and negative emotions people get. It makes people feel emotionless and very tired.

In other words, even if people are worried for a long time, people will get tired, and if people make a small reaction and give a small negative emotion $x$ repeatedly, people will get a big negative emotion to oneself. For example, it is extreme self-loathing, and extreme anger toward oneself and extreme disappointment toward oneself.

Therefore, if people are worried, I think it is better to set a short time and worry only for that time.

4. What to do if people don't get along well with people's surroundings

4.1. About reaction function

The probability density function of an individual's emotions is the same for everyone. However, some people worry about whether their joy and sadness are the same as others.

The probability density function of individual's and group's emotion in Figure 3 are $34 \%$ when emotion $x$ is between 0 and 1 (between emotionlessness, peace of mind, stability and normal happiness), 14\% when emotion $x$ is between 1 and 2 (very happy), and $2 \%$ when 2 or more (very, very happy), includes happy feelings. It is $50 \%$ in total. If it is $100 \%$, it will be doubled.

Think about this in a year. Most people feel "very, very happy" with the joy that occurs for about 14 days, which is $4 \%$ of 365 days. This is about once a month, which is about a payday. In addition, most people feel "very happy" with the joy that occurs for about 102 days, which is $28 \%$. This is the joy of having about two days a week, like giving something to oneself on Saturday and Sunday. What people feel on other days is "from emotionlessness, peace of mind, stability to normal joy." Negative emotions are the opposite of this. The degree of joy and sadness is as above.

\subsection{How to read the air}

If people don't get along well with people's surroundings, people may not be able to know what happened or what the other person was doing. It means that people cannot read the so-called air.

So, I explain an idea of how to read the air. First, we try to find out from the emotion processing itself. Here, the flux of emotion processing is defined by the following formula. Same as equation (15).

$$
Q=-\frac{1}{2} \frac{\partial p}{\partial x}
$$


Now, regarding the flux $Q_{y}$ of the emotion processing of the other party given by equation (24), if the event is dimensionless and the emotion has a dimension of $\mathrm{E}$, the dimension of $Q_{y}$ is $\left[\mathrm{ET}^{-1}\right]$. Note that $Q_{y}=\frac{d y}{d t}$ cannot be used here. $x$ is a random variable, and $Q_{y}=\frac{d W_{t}}{d t}$ from equation (10), but there is no time derivative of the Wiener process. Therefore, it is the emotional value per unit time, such as $Q_{y}=\frac{y}{\Delta t}$. If people want to know $Q_{y}$, people can understand the feelings of the other person. For this reason, people want to measure $Q_{y}$, but people need to know the emotions of the other person in order to do it directly, but people do not know that. Therefore, it is calculated from $Q_{y}=-\frac{1}{2} \frac{\partial p}{\partial y}$. $\partial p$, that is, $d p$, is also a change in the probability density of the other party, so people do not know. Also, people do not know $\partial y$, that is, $d y$, the minute change of the other party's emotions. Therefore, the flux $Q_{y}$ of the emotion processing of the other party cannot be measured directly or indirectly.

Therefore, another method is used to obtain the emotional value of the other party. The graph in Figure 3 shows the probability density function of most individual's and group's emotion. Take advantage of this.

First, slightly increase people's emotional value by $d x$. This will slightly increase the magnitude of people's reaction. Then the other party notices that people's reaction has increased, which changes the other party's emotions. If the other party's emotion is a positive sign, the reaction that is favorable to the other party has increased, so the minute change $d y$ of the emotion is in the direction of increasing, and the reaction is accompanied by a positive emotional change. If the other party's emotion is a negative sign, the reaction that is unfavorable to the other party has increased, so the minute change $d y$ of the emotion is in the direction of decreasing, and the reaction is accompanied by a negative emotional change.

Next, slightly decrease people's emotional value by $d x$. This will slightly decrease the magnitude of people's reaction. Then the other party notices that people's reaction has decreased, which changes the other party's emotions. If the other party's emotion is a positive sign, the reaction that is favorable to the other party has decreased, so the minute change $d y$ of the emotion is in the direction of decreasing, and the reaction is accompanied by a negative emotional change. If the other party's emotion is a negative sign, the reaction that is unfavorable to the other party has decreased, so the minute change $d y$ of the emotion is in the direction of increasing, and the reaction is accompanied by a positive emotional change. 
In other words, the sign of the other party's emotions can be understood from the change in the other party's behavior immediately after the person's reaction is slightly increased or slightly decreased. Immediately after people have a positive emotion and slightly decrease people's reaction and slightly decrease people's emotion, if the other party reacts with a negative emotional change, the other person and people have a positive emotion. Or immediately after people have a positive emotion and slightly increase people's reaction and slightly increase people's emotion, if the other party reacts with a positive emotional change, also the other party and people have a positive emotion. Immediately after people have a negative emotion and slightly increase people's reaction and slightly decrease people's emotion, if the other person reacts with a negative emotional change, the other person and people have a negative emotion. Or immediately after people have a negative emotion and slightly decrease people's reaction and slightly increase people's emotion, if the other party reacts with a positive emotional change, also the other person and people have a negative emotion.

On the contrary, immediately after people have a positive emotion and slightly decrease people's reaction and slightly decrease people's emotion, if the other party reacts with a positive emotional change, the other party has a negative emotion. Or immediately after people have a positive emotion and slightly increase people's reaction and slightly increase people's emotion, if the other party reacts with a negative emotional change, also the other person has a negative emotion. Immediately after people have a negative emotion and slightly increase people's reaction and slightly decrease people's emotion, if the other party reacts with a positive emotional change, the other party has a positive emotion. Or immediately after people have a negative emotion and slightly decrease people's reaction and slightly increase people's emotion, if the other party reacts with a negative emotional change, also the other person has a positive emotion.

As an example, suppose people are talking about something with positive emotions. By stopping the conversation or slowing down the pace of the conversation, people's reaction will be slightly decreased (people's emotions will be slightly decreased). Immediately after this, if the other party makes a negative emotional change such as a boring expression, it can be judged that the other party also has a positive emotion and wants to hear the story. On the other hand, if the other party makes a confused facial expression immediately after a slight increase in one's reaction (a slight increase in one's emotions), such as increasing the pace of conversation. It can be judged that the other party has a negative emotion and doesn't wants to hear the story.

"Reading the air" does not mean that there is any special number, but it is obtained by 
changing one's reaction and seeing the change in the behavior of the other party. People with high-functioning autism, such as Asperger's Syndrome, just didn't know how to do it.

With this remedy, people can read the air and understand the sign of the other party's emotion.

5. The true nature of positive and negative thinking

Mathematically think about "I can't do it because I'm a negative thinker," which people who are victims of bullying and moral harassment tend to fall into.

It is mathematically thought that positive thinking and negative thinking are the difference between easy memory of positive things and easy memory of negative things when calculating emotion $x$.

Now, I considered equation (4). The precondition at this time was that emotion $\mathrm{x}$ went to $x=0$. It turns out that it is a matter of the combination of 1 and -1 . With that in mind, we can see that the number of events that receive negative emotions and the number of events that receive positive emotions are actually the same. This is also clear from Figure 3.

In other words, in fact, there are the same number of positives and negatives, but people just choose randomly one or the other. Things have both good and bad sides. Even if people tend to memory negative things, it's a good idea to keep in mind that there are the same number of positive and negative things, and to look at the positive side without thinking too much about it.

I think some people say that my graph should not be as shown in Figure 3 because it is easy to think negatively. In the case of a one-dimensional random walk, it has the property that the probability of being in one of the positive and negative regions is greater than the probability of being in each of them. Therefore, there are people who are likely to receive negative emotions and people who are likely to receive positive emotions. Either way, it can be represented in the same Figure 3.

6. Is there really a one-dimensional random walk?

The precondition so far is that emotion processing is performed by a one-dimensional random walk. In other words, it is assumed that you have selected positive emotions and negative emotions with a probability of $\frac{1}{2}$. Many people think that they are really doing it with a probability of $\frac{1}{2}$, or that they have a high probability of choosing either positive or negative.

So, suppose the probability of choosing positive is $p$ instead of $\frac{1}{2}$. Then the probability 
of choosing a negative is $1-p$. At this time, the mean of the one-dimensional random walk is $n(2 p-1)$, and the variance is $4 n p(1-p)-n^{2}(2 p-1)^{2}$. The following equation is obtained by transforming the equation for variance.

$$
-4(1+n)\left(p-\frac{1}{2}\right)^{2}+1
$$

Since this value must be greater than or equal to 0 , the following conditions are obtained:

$$
\frac{1}{2}-\frac{1}{2 \sqrt{1+n}} \leq p \leq \frac{1}{2}+\frac{1}{2 \sqrt{1+n}}
$$

Therefore, the longer the emotion processing is performed, that is, when $n \rightarrow \infty$, the result is as follows.

$$
\frac{1}{2} \leq p \leq \frac{1}{2}
$$

In other words, like everyone who has done a long number of one-dimensional random walks, the probability must be $\frac{1}{2}$.

No, I haven't been bothered for a long time. In the first place, some people may say that $t \rightarrow 1$, that is, one second does not usually bother you. In Section 2.3, we showed that a discrete one-dimensional random walk is the same as a continuous Wiener process. The following relationships have been obtained between the one-dimensional random walk and the Wiener process. This is called Donsker's theorem. Here, $S_{\lfloor n t\rfloor}$ is the value obtained by randomly adding -1 and 1 for the maximum number of natural numbers up to $n t$.

$$
\lim _{n \rightarrow \infty} \frac{S_{\lfloor n t \mid}}{\sqrt{n}}=W_{t,} 0 \leq t \leq 1
$$

That is, for any short time between $0 \leq t \leq 1$, the one-dimensional random walk converges on the Wiener process. You can do $\infty$ times of one-dimensional random walks. You can choose $\infty$ times, and if you feel both positive and negative emotions, then equation ( 27) is satisfied.

In other words, in my model, a person who can feel both positive and negative emotions repeat a one-dimensional random walk with a probability of $\frac{1}{2}$. If the probability is not $\frac{1}{2}$, then the variance cannot be obtained, so statistical processing cannot be performed, and the probability density function of emotions with positive and negative emotions cannot be obtained. People can only have one of the emotions. If people can feel both emotions, people don't have to worry that people are different from a normal, neurotypical person from the probability density function of people's emotions. People can't build relationships well only because people don't know how to read the air. 
7. How to deal with your character

Again, in my mathematical model, if we remove the superficial part of the reaction, then every human being has the same probability density function of emotion. The only difference is how one reacts. Both popular and disliked people have the same probability density function of emotion.

Therefore, when people face people's character and want to correct people's character, people use the fact that people's character appears in the reaction, not your character. People will think about changing the reaction, that is shown when the event $X$ and emotion $x$ are determined like a dictionary, to the reaction you like. Individuals cannot influence the reactions that have occurred and emotions that have generated, but I think that individuals can change how they react. The probability density function of an individual's emotion (be created by one's character) is statistically determined and is the same for everyone. People can't fix it. Rather, people don't have to fix it. Is it easy to think negatively? Do people have to think positively? People with negative thoughts and people with positive thoughts have the same probability density function of emotion after all. It's statistically determined, so people can't fix it themselves.

Therefore, people will think about changing the reaction, that is shown when the event $X$ and emotion $x$ are determined like a dictionary, to the reaction you like. You dare to avoid the reaction that was supposed to determine event $X$ and emotion $x$ as before. When that happens, people will accumulate the experience that problems do not actually occur. By doing so, people will gradually change the reaction that was supposed to determine event $X$ and emotion $x$ to a reaction that people think is preferable to them. Doing so will fix the reaction.

Repeat this for various combinations of events $X$, emotions $x$, and reactions that people do not like. By doing so, I think that the superficial reaction will change to something that is favorable to them.

\section{How to deal with one's reaction}

In my mathematical model of event function, every human has the same probability density function. Also, if people show an exaggerated reaction, it is easy to judge that people have emotions with a large absolute value. On the contrary, if people show a calm reaction, it is easy to judge that they have emotions with a small absolute value. $x=0$ means a situation of convincing, reassuring, stable, and emotionless.

From these facts, it can be seen that when there is any reaction, it is better to calm down and take a small action before reacting. As a result, the other party feels at ease. 
Equation (23) also shows that it is good not to repeat the same action many times in a short period of time.

In other words, it is mathematically not good to think that people have done something very sorry to the other person and try to continue to apologize in a loud voice over and over again. Again, it's a good idea to calm down and take smaller actions before reacting anyway.

9. About the reaction when a big event occurs

Think about a big event, such as a depression caused by multiple actions or behaviors that cannot be handled, a big accident or illness, or a big natural disaster.

In this case, think of a big event as a collection of small events. In the case of depression, each word and action correspond to it. Even a big accident or illness is a combination of various events such as physical pain, surgery, and the need for money. So is a big natural disaster.

It is mathematically thought that the emotions that arise from these small events are added as in equation (23), causing rejection, neglect, and annoyance until depression.

If people are depressed, escape from the situation. In the case of a big accident, illness or big natural disaster, spend more time not thinking about it. By doing so, I think it would be better to make the magnitude of the event smaller than equation (23).

\section{Applications}

I have shown the mathematical model of the psychological aspects of human and have shown as application examples of the model, how to read the air and how to deal with one's character and how to show one's reaction and what to do for events which have a large magnitude. The same is said for cognitive behavioral therapy for these examples (Beck, 2020). The cognitive behavioral therapy is based on the results of clinical trials in many people, meaning that the results from the proposed model are the same as those that hit a lot of data. In other words, it can be said that the proposed mathematical model can express the psychological aspects of real human.

In addition, the proposed mathematical model can be simulated on a computer. This can give the computer the psychological aspects of human.

\section{Conclusion}

I considered the psychological aspects of human beings using the probability density function of emotions generated by emotional processing of events. As a result, it was found that the probability density function of the emotion of individuals and groups are 
expressed by the same equation (4), which is why the group is established. I also analyzed that the cause of bullying and moral harassment is that people who do not understand how to read the air are divided into those who attack and those who are attacked. I also thought mathematically about how to deal with people who are not familiar with their surroundings and who are suffering from bullying and power harassment. People don't get used to the surroundings well, and people are bullied because they don't know how to read the air, so they are floating because they are reacting differently from the surroundings, and the bullying and moral harassment perpetrators make a reaction that they find interesting. As a countermeasure, I also proposed how to read the air, as to what the ratio of positive and negative emotions in the probability density function of emotion is. Also, since their probability density function created by character is statistically determined and cannot be changed, I proposed a way that change their character by changing the reaction they show. Also, I proposed if people react the other party, it is better to calmly react small and not repeat. Finally, I talked about what to do if a big event happens.

\section{References}

Beck, S. J. (2020). Cognitive Behavior Therapy, Third Edition: Basics and Beyond. The Guilford Press.

Bolger, N., Zee, K. S., Rossignac-Milon, M., \& Hassin, R. R. (2019). Causal processes in psychology are heterogeneous. Journal of Experimental Psychology: General, 148(4).

Burigana, L., \& Vicovaro, M. (2020). Algebraic aspects of Bayesian modeling in psychology. Journal of Mathematical Psychology, Vol.94.

Busemeyer, J. R., \& Diederich, A. (2002). Survey of decision field theory. Mathematical Social Sciences.

Busemeyer, J. R., \& Townsend, J. T. (1993). Decision Field Theory: A dynamic cognition approach to decision making. Psychological Review.

Chamberlin, S. A. (2019). Affect in Mathematical Modeling. Springer.

Cox, G. E., \& Criss, A. H. (2020). Similarity leads to correlated processing: A dynamic model of encoding and recognition of episodic associations. Psychological Review, $127(5)$.

Hancock, T. O. (2019). Travel behaviour modelling at the interface between econometrics and mathematical psychology. $\mathrm{PhD}$ thesis, University of Leeds.

Hancock, T. O., Hess, S., \& Choudhury, C. F. (2019). Theoretical considerations for a dynamic model for dynamic data: bridging choice modelling with mathematical 
psychology. International Choice Modelling Conference 2019.

Neufeld, R. W., \& Cutler , C. D. (2019). Potential Contributions of Clinical Mathematical Psychology to Robust Modeling in Cognitive Science. Computational Brain \& Behavior, Vol. 2.

XIN, X., LI, Y., BI, Y., \& YAN , B. (2019). Quantum decision-making model based on equate-to-differentiate method: Explanation for the disjunction effect in prisoner's dilemma. Acta Psychologica Sinica, Vol. 51. 